\title{
Impact of a Patient Support Program on Patient Beliefs About Neovascular Age-Related Macular Degeneration and Persistence to Anti-Vascular Endothelial Growth Factor Therapy
}

This article was published in the following Dove Press journal:

Patient Preference and Adherence

\section{Andrew Chang' \\ John Stokes ${ }^{2,3}$ \\ Lindy Priestman ${ }^{4}$ \\ Connor Holmes ${ }^{4}$ \\ Peter Said ${ }^{5}$}

'Sydney Retina Clinic, Sydney Institute of Vision Science, Save Sight Institute, University of Sydney, Sydney, New South Wales, Australia; ${ }^{2}$ Inservio, Sydney, New South Wales, Australia; ${ }^{3}$ Department of Biomedical Sciences, Charles Sturt University, Orange, New South Wales, Australia; ${ }^{4}$ Atlantis Healthcare Pty Ltd, Sydney, New South Wales, Australia; ${ }^{5}$ Medical Affairs, Bayer Australia Ltd, Sydney, New South Wales, Australia
Correspondence: Andrew Chang Sydney Retina Clinic, 187 Macquarie Street, Sydney, New South Wales, 2000, Australia

Tel + 61292213755

$\mathrm{Fax}+61292211637$

Email achang@sydneyretina.com.au
Purpose: This study aimed to compare persistence between patients prescribed intravitreal aflibercept (IVT-AFL) for neovascular age-related macular degeneration (nAMD) in Australia enrolled on a patient support program (PSP) with that of a sample of patients from the Australian Pharmaceutical Benefits Scheme (PBS) dataset (10\% PBS sample); explore predictors of persistence; describe changes in patient beliefs over the course of their enrollment in a PSP for patients treated with IVT-AFL for nAMD; and assess patient satisfaction.

Participants and Methods: Participants prescribed IVT-AFL for the treatment of nAMD were invited to participate in the PSP. The PSP provided tailored support to patients through provision of a welcome pack, structured telephone calls, and information booklets. Persistence was defined in the PSP as the time from the start date in the program, until discontinuation from the program; and as the time from initial prescription until 6-months after the date of last prescription in the $10 \%$ PBS set. Persistence on the program and risk of discontinuation were modeled using Kaplan-Meier methods and Cox proportional hazards. In addition, persistence was compared between patients on the PSP and a 10\% PBS sample of patients prescribed IVT-AFL for nAMD.

Results: Persistence on treatment at 24 months was significantly higher in patients enrolled on the PSP compared to the PBS cohort $(88 \%$ vs $64 \%, \mathrm{p}<0.05)$. The risk of discontinuation in patients enrolled on the PSP was higher in patients identified at screening as "high-risk", those who were younger, or those with significant distance to travel for treatment. During the PSP, patients reported significant increase in their belief that they had control over their condition $(6.1 \pm 3.5$ to $6.8 \pm 3.7 ; \mathrm{p}=0.0034)$ and a reduction in concerns about treatment. Satisfaction with the PSP was high.

Conclusion: Patients provided with access to a PSP showed better persistence on treatment and improved beliefs about nAMD disease and its treatment compared to those in the PBS sample. Improved persistence rates may translate into better outcomes for the patient and the healthcare system, however, further research is required to determine which elements of the program are most beneficial, particularly to those at high risk of discontinuation.

Keywords: intravitreal aflibercept, patient satisfaction, illness perceptions, medication beliefs, neovascular macular degeneration, persistence

\section{Plain Language Summary}

- Neovascular age-related macular degeneration (nAMD for short) is an eye condition that affects central vision.

o In nAMD abnormal blood vessels grow at the back of the eye and leak fluid. 
- Aflibercept (known as Eylea ${ }^{\circledR}$ ) prevents blood vessels from growing abnormally by blocking vascular endothelial growth factor. To maintain vision improvements, it is important for people with nAMD to continue aflibercept injections.

- Researchers wanted to understand if a patient support program in Australia called SmartSight helped people with nAMD to continue treatment.

- SmartSight includes tips for managing nAMD, information booklets, and telephone calls with a nurse.

- Researchers compared results from 3573 people with nAMD who took part in SmartSight with 3177 people with nAMD who did not take part in the program.

- After 2 years, people who took part in SmartSight were more likely to continue aflibercept than those who did not take part.

- People felt more positive about treatment after taking part in the SmartSight program.

- SmartSight may help people with nAMD to continue aflibercept injections and have a more positive view of their condition and treatment. This could motivate people to attend regular eye injection appointments to help them maintain their vision.

\section{Introduction}

Globally, age-related macular degeneration (AMD) is estimated to affect up to 8.8 million people ${ }^{1}$ and is one of the leading causes of blindness and severe vision loss in Australia. ${ }^{2,3}$ The majority of untreated patients present with the dry form of the disease, progressing to wet, or neovascular, AMD (nAMD) in $10-20 \%$ of cases. ${ }^{4}$ It was estimated that in 2010 in Australia, there were approximately 107,000 people with nAMD affecting both eyes and that this would increase to over 215,000 in 2030 in the absence of effective prevention and treatment. ${ }^{5}$

Patients with nAMD who experience vision loss suffer significant psychological impact, with the incidence of depression being five times higher than in the general population. ${ }^{6}$ In addition, nAMD has been shown to have a negative impact on a patient's quality of life, including challenges with mobility, face recognition, day-to-day tasks, and self-care. ${ }^{7,8}$ Patients with nAMD require urgent referral to an ophthalmologist for treatment. ${ }^{9}$ nAMD is a chronic condition requiring long-term treatment. The wide introduction of anti-vascular endothelial growth factor (anti-VEGF) therapy has resulted in improvement and maintenance of visual function in the vast majority of patients with nAMD. ${ }^{10,11}$ After three initial monthly injections of the anti-VEGF agent, treatment is administered by intravitreal injection every 4 to 8 weeks, or most commonly, a treat and extend protocol (where treatment intervals are extended or reduced as long as the macula remains dry, or the fluid is stable and the vision is stable or unaffected). ${ }^{12-14}$ Despite effective advances in treatment, adherence to this long-term treatment regimen is challenging, and high rates of discontinuation are noted in real-world cohorts of patients with nAMD. ${ }^{15-17}$ In Australia, treatment of nAMD is subsidized, both in terms of procedural costs via the Medicare Benefits Schedule (MBS) and drug costs via the Pharmaceutical Benefits Scheme (PBS). The reimbursed cost to the patient of single prescriptions of aflibercept is capped at $\sim$ AUD\$41 per $4 \mathrm{mg}$ vial (2020 prices) for a general patient, and concessional patients pay considerably less. Concessional patients are those for whom a higher level of Government reimbursement for medication is available (eg Pensioners and Veterans). In Australia, persistence to anti-VEGF treatment for nAMD declines over time, to approximately $70 \%$ after 2 years, $50 \%$ after 5 years, and $40 \%$ after 6 years. ${ }^{18}$ Lack of persistence to therapy is associated with poor outcomes. ${ }^{19,20}$

The levels of non-persistence to intravitreal aflibercept (IVT-AFL) in real-world clinical settings were not observed in the randomized clinical trials for antiVEGFs, probably reflecting stricter follow-up protocols, fully subsidized access to therapy, and a greater level of engagement by patients in controlled trials. Some patients achieve robust gains in visual acuity after three initial monthly injections ${ }^{21,22}$ and may consider themselves cured and that they do not require further treatment. Others may not see any improvement in vision over time and conclude their treatment is "not working". Patient persistence to treatment may be affected by injection fatigue, a misconception that ongoing treatment is unnecessary, or concerns about their treatment. ${ }^{6,23}$ Patients may also be unwilling to continue treatment because of expenses, such as clinic diagnostic and procedural costs, drug costs, ${ }^{5,24,25}$ lost days of work/income for the patient and their caregiver, and other costs associated with their visit including parking or transport. Thus, in the real-world 
setting, persistence to treatment continues to decline over time. $^{18}$

One strategy that may increase patient persistence to treatment is improved education of doctors, clinic staff, patients, and their caregivers, to be able to better support the patient in their treatment. ${ }^{26,27}$ Patients with nAMD have reported they were not provided with adequate information on $\mathrm{nAMD}$ or its treatment at the time of diagnosis or at subsequent visits. ${ }^{28}$ In a 2010 survey, more than one in five patients with nAMD changed doctors because of a lack of communication, specifically around the costs of treatment and written information on treatment and its side effects. $^{5,19}$ To effectively communicate with a patient, it is important to understand the patient's beliefs and treatment awareness, their opinion on the necessity of treatment, and their concerns. ${ }^{26}$

Patient support programs (PSPs) have the potential to provide support to patients to improve persistence to treatment, which may result in clinical and humanistic benefits, and reduced healthcare utilization. ${ }^{29}$ To investigate this, we assessed the outcomes of patients enrolled in the SmartSight ${ }^{\text {TM }}$ PSP, introduced in 2012. Following World Health Organization guidance on adherence to long-term therapies in a general sense, ${ }^{30}$ the PSP supports patients receiving treatment with IVT-AFL through patient education, behavioral modification, and telephone follow-up over a 24-month period. Given that a global study reported that $82 \%$ of patients with nAMD needed caregiver support, caregivers are also supported through the PSP, to reduce the significant burden on caregivers who support patients with nAMD. ${ }^{31}$ The program is largely facilitated by clinic staff, including orthoptists and ophthalmic assistants including nurses.

The aims of the study were to describe changes in patient beliefs regarding nAMD and its treatment over the course of their enrollment; to explore predictors of persistence; to compare persistence of patients enrolled on the PSP with that of a general sample of patients prescribed IVT-AFL for nAMD in Australia; and to assess patient satisfaction with the PSP.

\section{Participants and Methods \\ Participants}

During the period December 2012 to May 2020, patients prescribed IVT-AFL by their physician for treatment of nAMD were invited to participate in the PSP by their physician ("opt in"). Eligible patients provided written informed consent to participate in the PSP and to provide aggregated data for publication purposes. Due to the type of data gathered, formal ethics committee assessment was not required in accordance with the Australian National Health and Medical Research Council's (NHMRC's) National Statement for the Ethical Conduct of Research in humans. ${ }^{32}$ Since 2012, the PSP has enrolled more than 4000 patients.

\section{Patient Support Program}

The content of the program was adapted throughout its course of delivery, based on direct feedback from patients. At launch, the PSP was designed with limited goals, focusing on IVT-AFL persistence to 3 months. It was supported by an orthoptist with little structure to call frequency and content. At this time, patients were provided with a welcome pack, including an Amsler grid pad for daily eye checks, a calendar with appointment reminder stickers, a resources booklet, and a magnifier. Over the course of 12 months, they received a safety booklet, a diet booklet, and a lifestyle booklet. Patients were supported with inbound phone calls by an orthoptist that included education on safety, diet, and lifestyle. In 2014, the PSP was adapted, using health psychology principles, to include screening calls designed to provide a tailored approach to patients, as well as surveys to support a more structured and individualized program, and was extended from 12 to 24 months to further address identified persistence issues. In addition to the information booklets provided in the first year, patients received two newsletters in the second year. Calls from an ophthalmic registered nurse were provided on enrollment, and at 3,6, $9,12,15,21$, and 24 months. The call at 6 months was provided to those in the high-risk group only (see below for risk screening).

A team of health psychologists, responsible for delivery of the program, designed a risk screening tool that segmented patients into a "high-risk" or "low-risk" journey (where risk was based on likelihood of discontinuation). Risk screening was on the basis of patient beliefs about nAMD and its treatment, which were measured using questions adapted from validated health psychology questionnaires (Brief Illness Perception Questionnaire [BIPQ] $]^{33}$ and Beliefs about Medicines Questionnaire $[\mathrm{BMQ}]^{23}$ ). Measurement questionnaires related to concerns about treatment, necessity of the intervention, illness beliefs, self-efficacy, consequences, 
knowledge, and intention. These constructs were selected as they have been shown to be key predictors of adherence across multiple disease states ${ }^{34,35}$ and are modifiable when targeted with appropriate interventions. Patients were asked to self-report their score on a scale of 0-10 for each item with a cut-off of 5 and above or 5 and below indicating additional support was needed, depending on the question. For example, patients were considered "high risk" if they indicated a score between 5 and 10 for concerns about treatment, or if they scored $0-5$ on the question asking them to rate how necessary they considered their treatment to be. Any patient who was identified as having a score that indicated potential poor persistence on any of the questions was allocated to the high-touch journey. While patients in both journeys received personalized interventions based on their responses to the questionnaires, the high-risk patients received a (single) additional nurse call. Additional calls were scheduled if it was identified during the programmed calls that patients needed further support. Patients were also encouraged to use inbound calls to the program, if necessary. Inbound calls were available via a toll-free number during business hours (9 am to 5 pm, Monday to Friday).

Questionnaires for risk assessment were administered to patients at baseline (entry to the program), 12, and 24 months; and questionnaires for measurement were administered at baseline, 3, 12, and 24 months. However, patients remained in their original (baseline) assigned risk group even if their risk status changed over time.

Patient satisfaction with the PSP (as opposed to satisfaction with treatment per se) was assessed at 24 months on a 0 to 10 scale (with 10 indicating higher satisfaction). Participants were asked about the program overall ("Thinking about the program overall, please indicate on a scale of 0 to 10 how supportive you found it"), and also about their satisfaction with each component of the PSP ("We are interested in how helpful you have found each element of the program: welcome pack, nurse calls: inbound; nurse calls: outbound, and booklets"). They were asked to rate their likelihood of recommending the program to other patients

How likely are you to recommend the support you have received from the patient support program to a friend, family member, or colleague with nAMD, who is being treated with IVT-AFL?

or to their doctor
How likely are you to recommend the support you have received from the patient support program to your doctor?

The last question was only introduced in February 2019, so only a small sample is available for analysis.

Patients were assigned a carer in some cases. These carers provided consent and could take calls on behalf of the participant if the participant was unable to because of language, physical, or location issues. Carers were typically children of the participant but may have been siblings or close friends. Participants could decide whether they wanted information sent to themselves or their carer, or to both. Carers also received an information booklet and could call the toll-free number if they had questions or concerns.

Persistence to the PSP was captured via self-reporting at every nurse call over the 24-month period. Patients who were no longer on treatment or who were uncontactable (lost to follow-up) were considered to have ceased treatment with IVT-AFL.

For further details on the operation and design, please contact the authors.

\section{0\% PBS Sample}

A de-identified sample of patients' data was extracted from the $10 \%$ PBS dataset, used with permission from Services Australia. The $10 \%$ PBS sample dataset is a standardized, longitudinal, unit-record extract containing all PBS medicine dispensing data for a random 10\% sample of Australians. ${ }^{36}$ Patients with nAMD were identified within the PBS dataset by the IVT-AFL PBS item code 2168D (patients with subfoveal choroidal neovascularization due to AMD). As this is an unselected group of patients with nAMD, it is possible that a proportion of patients enrolled in the PSP $(n=3054)$ might also be included in the $10 \%$ PBS sample.

\section{Statistical Methods}

All data were de-identified.

Persistence to IVT-AFL for patients in the $10 \%$ PBS dataset was defined as the time in consecutive days from the date of initial prescription for IVT-AFL until the date of discontinuation, that is, the date of the last prescription for IVT-AFL after which there is no additional prescription for IVT-AFL for a period of 6 months. This period was chosen as we assumed that at patient who had not filled their prescription for a period of 6 months would likely have ceased treatment, even if they were being 
treated under a "treat-and-extend" regime. Persistence to IVT-AFL on the PSP program for this analysis was defined as the time in consecutive days from the imputed IVT-AFL start date to the discontinuation from the PSP. Imputation of the IVT-AFL start date was necessary as patients did not always commence in the PSP immediately upon commencement of IVT-AFL. The IVT-AFL start date for PSP patients was imputed as the average time between starting IVT-AFL and starting in the PSP (given patients had already persisted to this point) and was imputed as 44 days; and the inferred stop date for IVT-AFL in the PSP to be 2 months less than the persistence to the program. This was considered more conservative than using the selfreported IVT-AFL start and stop dates from within the PSP. Persistence to IVT-AFL was estimated using KaplanMeier methods. Differences in persistence between patients on the PSP compared with those in the $10 \%$ PBS dataset were explored using relative risks of persistence at 24 months.

For within-program IVT-AFL persistence (that is, comparisons by year, or for predictors of persistence), persistence on IVT-AFL among patients enrolled in the PSP was defined as the time in consecutive days from the date of initial injection of IVT-AFL until the time of discontinuation of IVT-AFL within the PSP. Persistence to IVT-AFL was estimated using Kaplan-Meier methods. Patient characteristics associated with treatment persistence were explored using a Cox proportional hazards model. The following variables were included in the model: patient risk (low vs high), age (51-60, 61-70, 71-80, and over 80 years), state of residence, unilateral vs bilateral, and switch versus naïve. Variables found to be insignificant within the model were removed in the final model (number of eyes affected and switch vs naïve).

Differences in participant beliefs or satisfaction by age, sex, state, patient risk, treatment status (switch vs naïve), or number of eyes affected were explored using the Friedman test for repeated surveys, and the Wilcoxon signed rank test (to assess for differences between each pair of surveys). Analyses of changes in persistence over time (using Log rank tests) and patient beliefs and satisfaction (as outlined above) were completed by Atlantis Healthcare (Australia) Pty Ltd using the " $r$ " statistical programming language. All data are presented as mean \pm standard deviation, unless otherwise specified. Values of $p<0.05$ were considered statistically significant.

\section{Results}

\section{Participants}

Between December 2012 and November 2019, 3573 patients from across Australia had participated in the PSP. Participants in the PSP were comparable to those in the $10 \%$ PBS dataset. The mean \pm SD age of PSP participants was $84.3 \pm 8.9$ years, and two-thirds were female. Participant demographics are shown in Table 1. The mean \pm SD age of patients in the $10 \%$ PBS sample was $78.0 \pm$ 12.3 , with $54 \%$ female. The comparison between the $10 \%$ PBS sample and the PSP sample included 3177 patients from the 10\% PBS sample and 3054 from the PSP (analysis conducted in December 2019). For all other analyses, the full number of patients who had participated in the PSP at the time of the additional analyses ( $\mathrm{n}=3573$ patients) were included (analysis conducted May 2020, hence increased PSP numbers at that time).

\section{Persistence}

We compared treatment persistence in the 10\% PBS data sample $(n=3177)$ with persistence of $3054 / 3573$ patients enrolled in the PSP. Patients enrolled in the

Table I Patient Support Program Participant Demographics

\begin{tabular}{|l|l|}
\hline Demographics & $\%$ \\
\hline Sex & \\
Female & 61 \\
Male & 38 \\
Unknown & 2 \\
\hline Age group & \\
$51-60$ years & 1 \\
$61-70$ years & 7 \\
$71-74$ years & 7 \\
$75-79$ years & 13 \\
$80-84$ years & 20 \\
$85-89$ years & 23 \\
90 years or over & 26 \\
Unspecified & 3 \\
\hline With a nominated carer & 22 \\
\hline Eyes affected & \\
One & 23 \\
Two & 71 \\
Unknown & 6 \\
\hline Treatment status & \\
Treatment-naive & 50 \\
Switch & 32 \\
Unknown & 18 \\
\hline
\end{tabular}




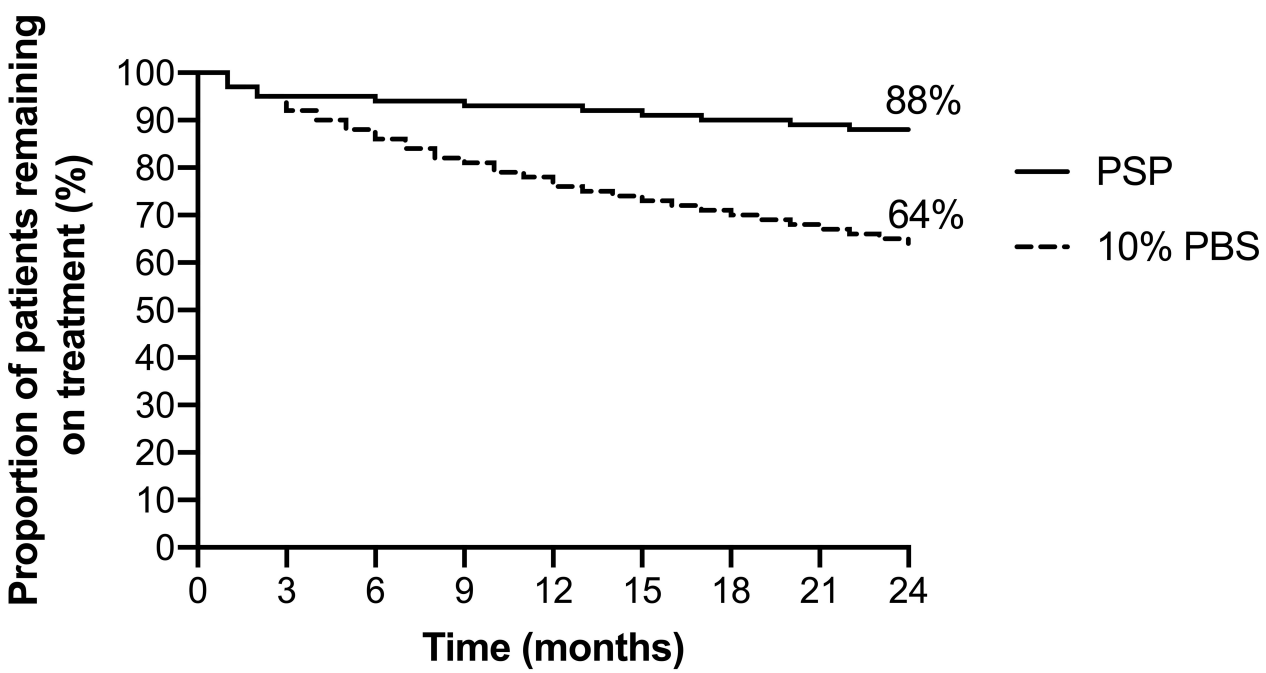

$\begin{array}{llllllllll}\text { At risk } 0 & 3 & 6 & 9 & 12 & 15 & 18 & 21 & 24 \\ \text { PBS } & 3177 & 2313 & 1706 & 1312 & 1065 & 886 & 753 & 645 & 547 \\ \text { PSP } & 3054 & 2724 & 2606 & 2485 & 2391 & 2224 & 2021 & 1812 & 1634\end{array}$

Figure I Treatment persistence in patients enrolled in the PSP vs the 10\% PBS data set.

Abbreviations: PBS, Pharmaceutical Benefits Scheme; PSP, patient support program.

PSP were more likely to be adherent to treatment (Figure 1). At 12 months, persistence was $76 \%$ in the PBS cohort and $93 \%$ in the PSP cohort. By 24 months, persistence was $64 \%$ and $88 \%$, respectively. Based on these rates, we estimate there are approximately 2.0 additional injections over a 24-month period are given to patients involved in the PSP compared to those who were not.

Persistence to IVT-AFL improved over the 6 years of the program, with 2 -year persistence being $71 \%$ at the start of the program and rising to $89 \%$ for those enrolling in 2018 (Table 2). The risk of ceasing treatment was two times higher in patients categorized as high risk compared with those categorized as low risk, suggesting the screening tool was an effective way of allocating patients into high-risk or low-risk treatment journeys (Table 3). Those with significant distance to travel to their healthcare practitioner $(>100 \mathrm{~km})$ were $52 \%$ less likely to be adherent than those located within $20 \mathrm{~km}$ (Table 3). Based on these, we allocated patients to a low $(n=485)$, medium $(n=897)$, or high $(n=30)$ risk of non-persistence to treatment (Figure 2). Some differences were noted between the sub-group analysis of persistence and that of the program overall. This was due to only patients with fully completed data sets being included in the subanalysis.

\section{Participant Beliefs}

Over time, participant concerns with having IVT-AFL injections for their nAMD significantly decreased from baseline to 24 months, from $3.3 \pm 3.8$ to $1.9 \pm 3.2(\mathrm{p}<0.05)$; and patients' perceptions of whether or not IVT-AFL injections could help their nAMD significantly improved $(8.1 \pm 2.3$ to $9.0 \pm 2.1 ; \mathrm{p}<0.0001)$. Over time, participants felt they had more control over their nAMD $(6.1 \pm 3.5$ to $6.8 \pm 3.7$; $\mathrm{p}=0.0034$ ) and were coping significantly better at the end of the program $(8.5 \pm 2.2$ to $8.8 \pm 2.3 ; \mathrm{p}=0.00013)$.

There was no clinically significant change in the perceived need for injections over the PSP period $(9.4 \pm 1.7$ at

Table 2 Persistence to Patient Support Program by Year of Enrollment

\begin{tabular}{|l|l|l|l|}
\hline $\begin{array}{l}\text { Year of } \\
\text { Enrollment }\end{array}$ & $\mathbf{N}$ & $\begin{array}{l}\text { Persistence to Program } \\
\text { at 24 Months }\end{array}$ & $\mathbf{9 5 \%} \mathbf{~ C l}$ \\
\hline 2012 & 112 & $71.3 \%$ & $63.3-80.4 \%$ \\
\hline 2014 & 781 & $85.3 \%$ & $82.7-87.9 \%$ \\
\hline 2016 & 484 & $87.0 \%$ & $83.9-90.3 \%$ \\
\hline 2018 & $178^{\mathrm{a}}$ & $88.9 \%$ & $83.5-94.2 \%$ \\
\hline
\end{tabular}

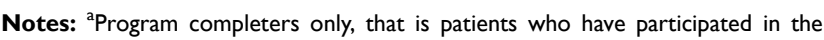
telephone call at 24 months. 2018 figures are for program completers only as the program is ongoing.

Abbreviation: $\mathrm{Cl}$, confidence interval. 
Table 3 Predictors of Persistence on Program from Cox Proportional Hazards Regression Model

\begin{tabular}{|c|c|c|c|c|c|}
\hline Patient Attribute & Regression Coefficient & Hazard Ratio & $p$ value & Cl (Lower 0.95) & Cl (Upper 0.95) \\
\hline nAMD high-risk patient ${ }^{a}$ & 1.469 & 4.34 & $<0.0001$ & 3.00 & 6.29 \\
\hline nAMD low-risk patient & 0.829 & 2.29 & $<0.0001$ & 1.55 & 3.39 \\
\hline Age range $6 I-70$ years $^{b}$ & -0.750 & 0.47 & 0.03 & 0.24 & 0.93 \\
\hline Age range $7 I-80$ years $^{c}$ & -0.904 & 0.40 & $<0.001$ & 0.22 & 0.75 \\
\hline Age range over 80 years $^{c}$ & -0.973 & 0.38 & $<0.01$ & 0.21 & 0.69 \\
\hline HCP distance over $100 \mathrm{~km}^{\mathrm{c}}$ & 0.482 & 1.62 & $<0.01$ & 1.15 & 2.28 \\
\hline
\end{tabular}

Notes: ${ }^{a}$ Reference category: patients treated prior to the introduction of risk stratification; ${ }^{b}$ reference category: $5 \mathrm{I}-60$ years; ${ }^{\mathrm{c}}$ reference category: $<20 \mathrm{~km}$.

Abbreviations: AMD, age-related macular degeneration; $\mathrm{Cl}$, confidence interval; HCP, healthcare professional; nAMD, neovascular age-related macular degeneration.

baseline, $8.8 \pm 2.7$ at 24 months; $\mathrm{p}<0.001$ ) or participants' confidence with being able to continue their injections ( 9.6 \pm 1.8 to $9.7 \pm 1.7$ at 12 months; $p=0.95)$. Note there was insufficient data to include 24-month outcomes for the confidence measure.

Patients with negative perceptions at baseline, as assessed by our risk assessment questionnaire, reported lower persistence than those with positive profiles (lowrisk vs high-risk hazard ratio, 0.829; $\mathrm{p}<0.0001$,
Supplementary Table 1), consistent with our initial stratification of patients into low-risk and high-risk groups.

\section{Patient Satisfaction}

Overall, patients were very satisfied with the PSP, with a mean overall satisfaction score of 8.3. Satisfaction was consistent across all patient types, regardless of patient age, sex, state of residence, risk status, treatment status, or number of eyes affected (Figure 3). The greatest

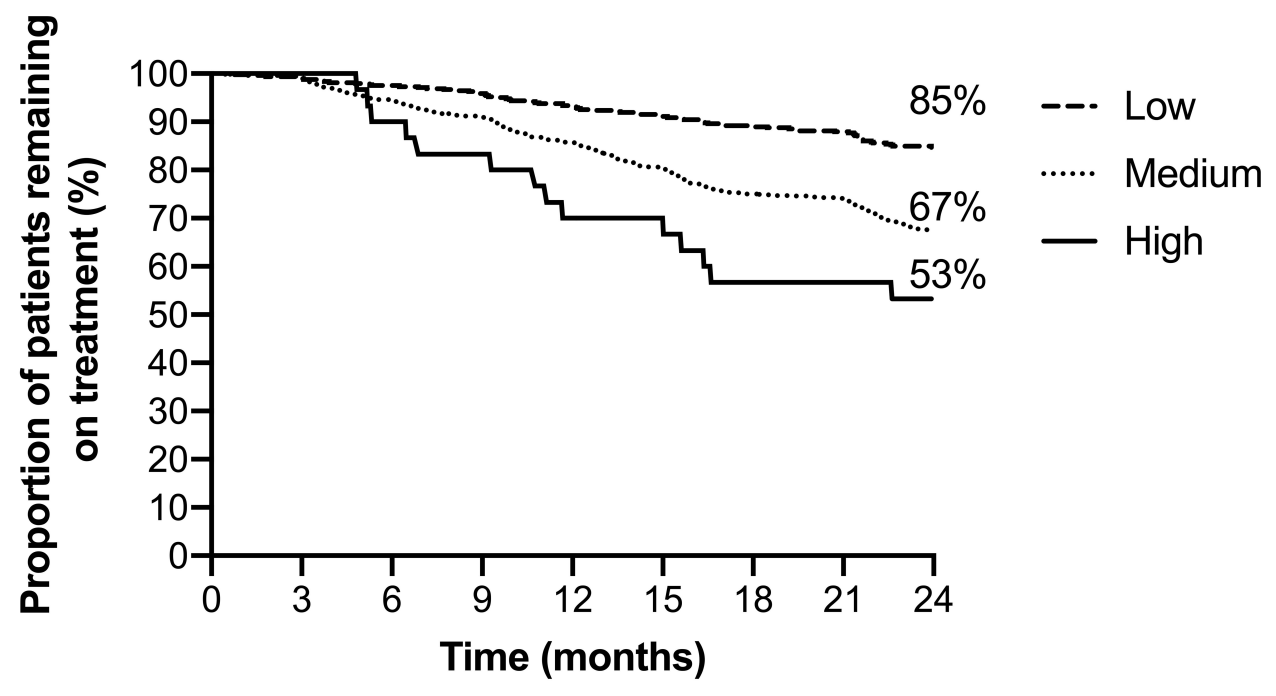

$\begin{array}{llllllllll}\text { At risk } & 0 & 3 & 6 & 9 & 12 & 15 & 18 & 21 & 24 \\ \text { Low } & 485 & 482 & 472 & 464 & 450 & 438 & 425 & 419 & 363 \\ \text { Medium } & 897 & 889 & 841 & 810 & 761 & 709 & 660 & 649 & 727 \\ \text { High } & 30 & 30 & 28 & 26 & 22 & 21 & 21 & 21 & 17\end{array}$

Figure 2 Persistence by risk category.

Notes: The risk stratification was based on combinations of the risk factors identified in the Cox proportional hazards regression model: that is, younger, high-risk patients, who live further from their HCP have a lower persistence than older, low-risk patients, who live less than $100 \mathrm{~km}$ from their HCP. Note there are differences in the number of patients in each risk category (low $(n=485)$, medium $(n=897)$ and high $(n=30)$ ).

Abbreviation: HCP, healthcare professional. 


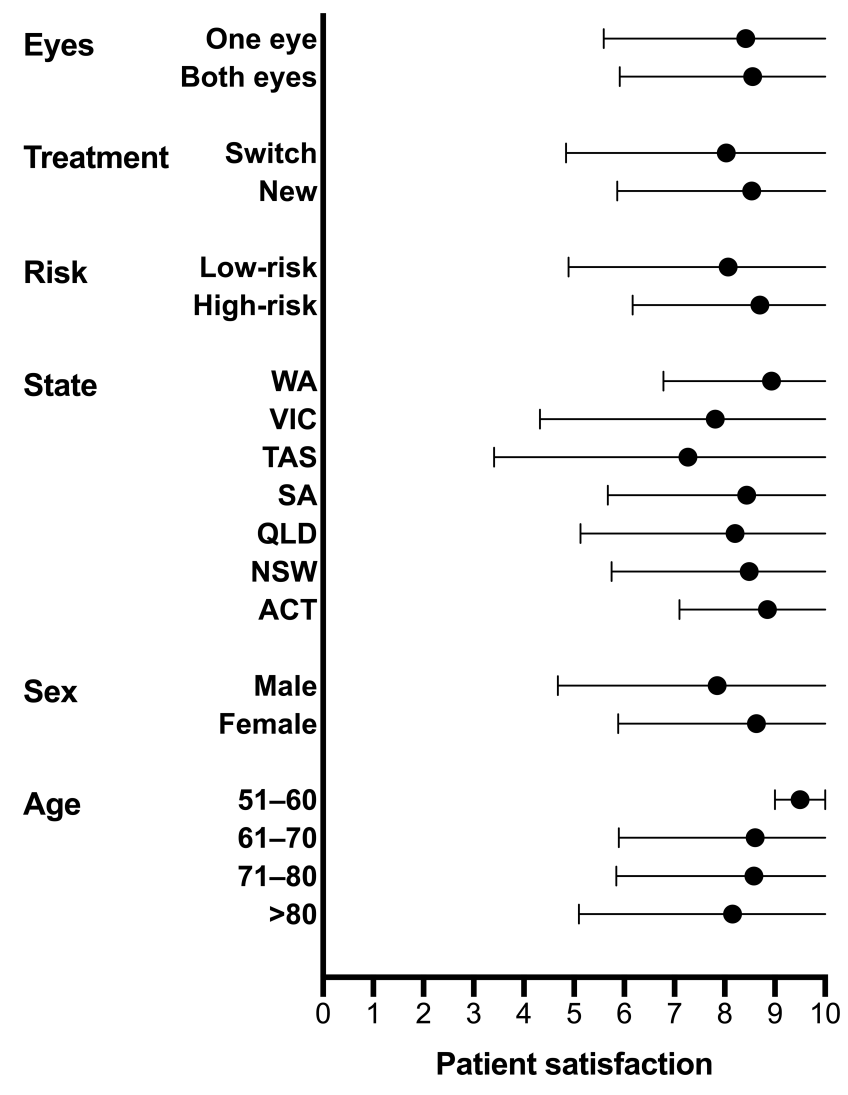

Figure 3 Patient satisfaction (mean $\pm S D$ ) with the patient support program. Note: Standard deviations are truncated at the upper level of patient satisfaction (I0).

Abbreviations: ACT, Australian Capital Territory; NSW, New South Wales; QLD, Queensland; SA, South Australia; SD, standard deviation, TAS, Tasmania; VIC, Victoria, WA, Western Australia.

satisfaction with the PSP was with inbound telephone calls (rated as $9.2 \pm 1.4$, Supplementary Table 2).

Patients reported several benefits of the program, including feeling that someone cared,

The wonderful thing that came into my life after the first injection was to be contacted [by] the SmartSight program. And to know that there was somebody there that was interested

that they felt encouraged,

I have been on the SmartSight program for at least 6 months. I find that very helpful where they ring up and they explain things and they keep you on track and they just keep you going

and that they understood why persistence to treatment was important,

I certainly will be staying on the program. I felt my eyes were so good I did ask my doctor 'Is it necessary for me to keep having it?' and he assured me it was, and also the SmartSight nurse did too, because you can lose the wonderful progress you've made.

\section{Discussion}

Given the importance of persistence to treatment with antiVEGF therapy to visual acuity outcomes in nAMD, ${ }^{37,38}$ it is extremely important that all avenues that can help improve persistence to treatment are explored. A meta-analysis of PSPs for chronic diseases in general supports the positive impacts on persistence to treatment, clinical and humanistic outcomes, and costs in healthcare utilization following their implementation. ${ }^{29}$ In this study, we have found significantly higher persistence in patients enrolled in the PSP compared with the general population of patients administered IVTAFL for nAMD. This reflects others' findings that complimentary PSPs have a favorable effect on patient persistence. ${ }^{6}$ The loss to follow-up rates in our general patient cohort (7\%) were higher than an Austrian study, which reported loss to follow-up of less than $3 \%$ in a group of patients with AMD who had universal health coverage. ${ }^{15}$ This may reflect the higher out-of-pocket costs and the number of injections given in Australia compared to Austria.

Patients predicted to have greater persistence included those with lower risk status based on screening questions, older age, and closer distance to the healthcare clinic. Importantly, our prescreening questionnaire appropriately stratified patients who were at greater risk of ceasing treatment; however, we were not fully able to support these patients. It may be valuable to explore ways to offer additional support to this high-risk group to increase their level of persistence. Patients require appropriate counseling at the start of a course of treatment to align expectations with perceived treatment outcomes in order to improve persistence to treatment. ${ }^{27}$ Perhaps this counseling was adequate for patients identified as low risk, but enhanced education and counseling is required for those considered high risk. Of concern, others have reported that just under $17 \%$ of patients with nAMD are not aware that their condition may result in vision loss or blindness, ${ }^{26}$ and a majority of patients hope that intravitreal therapy is only required temporarily. ${ }^{26}$ However, these attitudes can be modified over time with adequate education, as evidenced by the significant reduction in concerns about treatment from baseline to 24 months in our study.

As expected, greater patient distance from the healthcare professional (HCP) was identified as a risk factor for 
lack of persistence. This highlights the need to provide patients living outside metropolitan centers with as much support as possible to stay on treatment. An Australian survey conducted in 2010 noted that up to $20 \%$ of patients who live in small rural or remote areas have difficulties accessing treatment because of the distance required to travel. $^{39}$

Significant improvements in patient beliefs were observed over the course of the PSP. Concerns about treatment declined, with minimal change in attitudes towards the need for treatment. Whether these are causally related to the improved treatment persistence is unknown. Further, patient satisfaction with the PSP was high, and inbound calls were particularly valued. Thus, PSPs such as the one we have implemented might optimize the potential benefits of anti-VEGF therapy in nAMD by ensuring the quality use of medicines.

Our study has several limitations, including that it is based on observational data, and that discontinuation was based on self-report. We do not know whether those who chose to participate in the PSP differ from those who did not, and we have not adjusted for any potential confounding factors between the PSP and the 10\% PBS data sample. Further, a very small portion of patients on the PSP are likely to be represented within the $10 \%$ PBS data sample. Another potential limitation is that the program was extensively adapted in 2014 and the data includes patient responses from that earlier version of the PSP.

\section{Conclusion}

Patients provided with access to a PSP show better persistence and improved understanding about both nAMD disease and its treatment. Improved persistence rates can be expected to translate into better outcomes for the patient and the healthcare system; however, further research is required to determine which elements of the program are of most benefit, particularly for those at high risk of discontinuation. An extended plain language summary of this paper is available as Figure S1 in the supplementary text.

\section{Acknowledgments}

The authors thank Eric Chung from Prospection Pty Ltd for performing an earlier data analysis that prompted this research, Matthew Tucker from Atlantis Healthcare (Australia) Pty Ltd for performing PSP and PBS 10\% data analysis, and Rachelle Steele, BPharm (Hons), and Belinda Butcher, BSc (Hons), MBiostat, PhD, CMPP, of WriteSource Medical Pty Ltd, Sydney, NSW, Australia, for providing medical writing support (funded by Bayer Australia Pty
Ltd, Pymble, NSW, Australia, in accordance with Good Publication Practice guidelines). The authors thank Dawn Lobban at Envision Pharma Group for support in preparing the plain language summary of this paper (funded by Bayer Consumer Care AG, Basel, Switzerland). This study was funded by Bayer Australia Ltd.

\section{Disclosure}

AC reports consulting fees from Allergan, Novartis, Bayer, and Alcon, and involvement in clinical trials with Allergan, Novartis, Bayer, Alcon, Ophthotech, Opthea, Ionis, Oncobiologics, and Biofirst. JS reports no conflicts of interest. LP and $\mathrm{CH}$ are employees of Atlantis Healthcare (Australia) Pty Ltd who designed and delivered the SmartSight program. Bayer commissioned and sponsored the SmartSight program. PS is an employee of Bayer Australia Ltd, who manufacture Eylea (IVT-AFL). The authors report no other conflicts of interest in this work.

\section{References}

1. Flaxman SR, Bourne RRA, Resnikoff S, et al. Global causes of blindness and distance vision impairment 1990-2020: a systematic review and meta-analysis. Lancet Glob Health. 2017;5(12):e1221e1234. doi:10.1016/S2214-109X(17)30393-5

2. Taylor HR, Keeffe JE, Vu HTV, et al. Vision loss in Australia. Med J Aust. 2005;182(11):565-568. doi:10.5694/j.1326-5377.2005.tb06815.x

3. Foreman J, Keel S, McGuiness M, et al. Future burden of vision loss in Australia: projections from the national eye health survey. Clin Exp Ophthalmol. 2020;48(6):730-738. doi:10.1111/ceo.13776

4. Al-Zamil WM, Yassin SA. Recent developments in age-related macular degeneration: a review. Clin Interv Aging. 2017;12:1313-1330. doi:10.2147/CIA.S143508

5. Mitchell P, Deloitte Access Economics. Eyes on the Future: A Clear Outlook on Age-Related Macular Degeneration. 2011.

6. Stokes J, Chang AA. Adherence and persistence with anti-VEGF treatment for AMD. Mivision. 2016;114:51-55.

7. Bourla DH, Young TA. Age-related macular degeneration: a practical approach to a challenging disease. J Am Geriatr Soc. 2006;54 (7):1130-1135. doi:10.1111/j.1532-5415.2006.00771.x

8. Taylor DJ, Hobby AE, Binns AM, Crabb DP. How does age-related macular degeneration affect real-world visual ability and quality of life? A systematic review. BMJ Open. 2016;6(12):e011504. doi:10.1136/bmjopen-2016-011504

9. Optometry Australia. 2019 Clinical Practice Guide for the Diagnosis, Treatment and Management of Age-Related Macular Degeneration; 2019.

10. Schmidt-Erfurth U, Chong V, Loewenstein A, et al. Guidelines for the management of neovascular age-related macular degeneration by the European Society of Retina Specialists (EURETINA). Br J Ophthalmol. 2014;98(9):1144-1167. doi:10.1136/bjophthalmol-2014-305702

11. Mehta H, Tufail A, Daien V, et al. Real-world outcomes in patients with neovascular age-related macular degeneration treated with intravitreal vascular endothelial growth factor inhibitors. Prog Retin Eye Res. 2018;65:127-146. doi:10.1016/j.preteyeres.2017.12.002

12. Guymer RH, Markey CM, McAllister IL, et al. Tolerating subretinal fluid in neovascular age-related macular degeneration treated with ranibizumab using a treat-and-extend regimen: FLUID Study 24-month results. Ophthalmology. 2019;126(5):723-734. doi:10.1016/j.ophtha.2018.11.025 
13. Ohji M, Takahashi K, Okada AA, et al. Efficacy and safety of intravitreal aflibercept treat-and-extend regimens in exudative age-related macular degeneration: 52- and 96-week findings from ALTAIR: a randomized controlled trial. Adv Ther. 2020;37 (3):1173-1187. doi:10.1007/s12325-020-01236-x

14. Mitchell P, Souied EH, Midena E, Holz FG, Hykin PGW, Allmeier S. Efficacy of intravitreal aflibercept administered using treat-andextend regimen over 2 years in patients with neovascular age-related macular degeneration: 1-year ARIES results. Invest Ophthalmol Vis Sci. 2019;60(9):117.

15. Angermann R, Rauchegger T, Nowosielski Y, et al. Treatment compliance and adherence among patients with diabetic retinopathy and age-related macular degeneration treated by anti-vascular endothelial growth factor under universal health coverage. Graefes Arch Clin Exp Ophthalmol. 2019;257(10):2119-2125. doi:10.1007/s00417-019-04414-y

16. Obeid A, Gao X, Ali FS, et al. Loss to follow-up among patients with neovascular age-related macular degeneration who received intravitreal anti-vascular endothelial growth factor injections. JAMA Ophthalmol. 2018;136(11):1251-1259. doi:10.1001/jamaophthalmol.2018.3578

17. Boulanger-Scemama E, Querques G, About F, et al. Ranibizumab for exudative age-related macular degeneration: a five year study of adherence to follow-up in a real-life setting. $J$ Fr Ophtalmol. 2015;38(7):620-627. doi:10.1016/j.jfo.2014.11.015

18. Drug Utilisation Sub-Committee. Ranibizumab and Aflibercept: Analysis of Use for AMD, DMO, BRVO and CRVO. Canberra: Pharmaceutical Benefits Advisory Committee; 2018.

19. Vaze A, Fraser-Bell S, Gillies M. Reasons for discontinuation of intravitreal vascular endothelial growth factor inhibitors in neovascular age-related macular degeneration. Retina. 2014;34 (9):1774-1778. doi:10.1097/IAE.0000000000000173

20. Cohen SY, Mimoun G, Oubraham H, et al. Changes in visual acuity in patients with wet age-related macular degeneration treated with intravitreal ranibizumab in daily clinical practice: the LUMIERE study. Retina. 2013;33(3):474-481. doi:10.1097/ IAE.0b013e31827b6324

21. Dugel PU, Koh A, Ogura Y, et al. HAWK and HARRIER: Phase 3, multicenter, randomized, double-masked trials of brolucizumab for neovascular age-related macular degeneration. Ophthalmology. 2020;127(1):72-84. doi:10.1016/j.ophtha.2019.04.017

22. Heier JS, Brown DM, Chong V, et al. Intravitreal aflibercept (VEGF trap-eye) in wet age-related macular degeneration. Ophthalmology. 2012;119(12):2537-2548. doi:10.1016/j.ophtha.2012.09.006

23. Horne R, Weinman J, Hankins M. The beliefs about medicines questionnaire: the development and evaluation of a new method for assessing the cognitive representation of medication. Psychol Health. 1999;14(1):1-24. doi:10.1080/08870449908407311

24. Spooner KL, Mhlanga CT, Hong TH, Broadhead GK, Chang AA. The burden of neovascular age-related macular degeneration: a patient's perspective. Clin Ophthalmol. 2018;12:2483. doi:10.2147/OPTH.S185052

25. Spooner KL, Guinan G, Koller S, Hong T, Chang AA. Burden of treatment among patients undergoing intravitreal injections for diabetic macular oedema in Australia. Diabetes Metab Syndr Obes. 2019;12:1913. doi:10.2147/DMSO.S214098
26. Müller S, Ehlken C, Bauer-Steinhusen U, et al. Treatment of agerelated neovascular macular degeneration: the patient's perspective. Graefes Arch Clin Exp Ophthalmol. 2017;255(11):2237-2246. doi:10.1007/s00417-017-3739-1

27. Sii S, Aspinall P, Borooah S, Dhillon B. Exploring factors predicting changes in patients' expectations and psychosocial issues during the course of treatment with intravitreal injections for wet age-related macular degeneration. Eye. 2018;32(4):673-678. doi:10.1038/ eye. 2017.271

28. Mitchell J, Bradley P, Anderson S, Bradley C. Perceived quality of health care in macular disease: a survey of members of the Macular Disease Society. Br J Ophthalmol. 2002;86(7):777-781. doi:10.1136/ bjo.86.7.777

29. Ganguli A, Clewell J, Shillington AC. The impact of patient support programs on adherence, clinical, humanistic, and economic patient outcomes: a targeted systematic review. Patient Prefer Adherence. 2016;10:711-725. doi:10.2147/PPA.S101175

30. World Health Organization. Adherence to Long-Term Therapies: Evidence for Action. World Health Organization; 2003.

31. Varano M, Eter N, Winyard S, Wittrup-Jensen KU, Navarro R, Heraghty J. Current barriers to treatment for wet age-related macular degeneration (wAMD): findings from the wAMD patient and caregiver survey. Clin Ophthalmol. 2015;9:2243. doi:10.2147/OPTH.S92548

32. National Health and Medical Research Council, The Australian Research Council, Universities Australia. National Statement on Ethical Conduct in Human Research (2007) Updated 2018. Canberra: Commonwealth of Australia;2018.

33. Broadbent E, Petrie KJ, Main J, Weinman J. The brief illness perception questionnaire. $J$ Psychosom Res. 2006;60(6):631-637. doi:10.1016/j.jpsychores.2005.10.020

34. Foot H, La Caze A, Gujral G, Cottrell N. The necessity-concerns framework predicts adherence to medication in multiple illness conditions: a meta-analysis. Patient Educ Couns. 2016;99(5):706-717. doi:10.1016/j.pec.2015.11.004

35. Kucukarslan SN. A review of published studies of patients' illness perceptions and medication adherence: lessons learned and future directions. Res Social Adm Pharm. 2012;8(5):371-382. doi:10.1016/ j.sapharm.2011.09.002

36. Mellish L, Karanges EA, Litchfield MJ, et al. The Australian pharmaceutical benefits scheme data collection: a practical guide for researchers. BMC Res Notes. 2015;8(1):634. doi:10.1186/s13104015-1616-8

37. Kim JH, Chang YS, Kim JW. Natural course of patients discontinuing treatment for age-related macular degeneration and factors associated with visual prognosis. Retina. 2017;37(12):2254-2261. doi:10.1097/IAE.0000000000001494

38. Ramakrishnan MS, Yu Y, VanderBeek BL. Association of visit adherence and visual acuity in patients with neovascular age-related macular degeneration: secondary analysis of the comparison of age-related macular degeneration treatment trial. JAMA Ophthalmol. 2020;138(3):237-242. doi:10.1001/jamaophthalmol.2019.4577

39. Macular Degeneration Foundation. Barriers to Accessing Wet MD Treatment in Australia - a Patient Perspective. Macular Degeneration Foundation; 2010 


\section{Publish your work in this journal}

Patient Preference and Adherence is an international, peer-reviewed, open access journal that focusing on the growing importance of patient preference and adherence throughout the therapeutic continuum. Patient satisfaction, acceptability, quality of life, compliance, persistence and their role in developing new therapeutic modalities and compounds to optimize clinical outcomes for existing disease

states are major areas of interest for the journal. This journal has been accepted for indexing on PubMed Central. The manuscript management system is completely online and includes a very quick and fair peer-review system, which is all easy to use. Visit http:// www.dovepress.com/testimonials.php to read real quotes from published authors. 